\title{
VISUALIZACIÓN Y RECUPERACIÓN DE INFORMACIÓN EN LA WEB SEMÁNTICA
}

\author{
Jorge Morato, Sonia Sánchez-Cuadrado, Alejandro Ruiz-Robles y José- \\ Antonio Moreiro-González
}

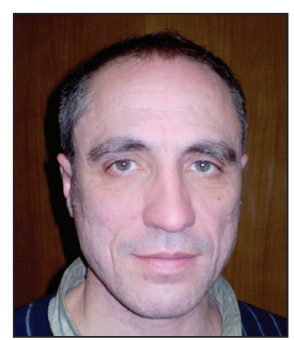

Jorge Morato es doctor en documentación y trabaja en el Departamento de Informática de la Universidad Carlos III de Madrid. Sus principales publicaciones están relacionadas con la utilización y construcción automática de tesauros y ontologías en la mejora de la recuperación de información y gestión del conocimiento.

http://orcid.org/0000-0002-7530-9753

Universidad Carlos III de Madrid, Departamento de Informática Avda. Universidad, 30. 28911 Leganés (Madrid), España jmorato@inf.uc3m.es

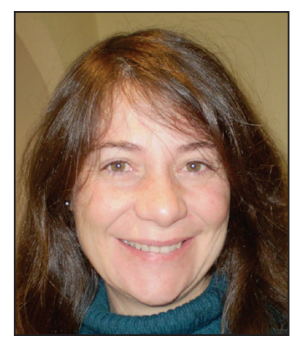

Sonia Sánchez-Cuadrado es doctora en documentación y ha sido profesora del Departamento de Informática de la Universidad Carlos III durante más de una década. Actualmente trabaja en sistemas de procesamiento del lenguaje natural en publicidad SEO. Su actividad investigadora está enfocada a la extracción de información, reconocimiento de patrones, procesamiento del lenguaje natural y sistemas de organización del conocimiento.

http://orcid.org/0000-0002-7722-1982

JOT Internet Media Gral. Ramírez de Madrid, 6-8. 28020 Madrid, España ssanchec@gmail.com

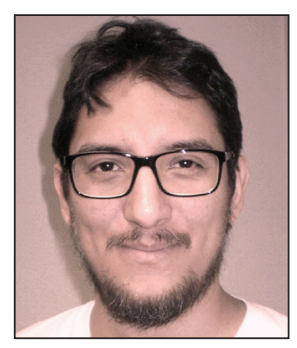

Alejandro Ruiz-Robles es máster en ciencia y tecnología informática por la Universidad Carlos III de Madrid (UC3M) y profesor a tiempo completo por la Universidad de Piura (Perú). Actualmente cursa el doctorado en la UC3M y su principal área de investigación es la gestión del conocimiento. http://orcid.org/0000-0002-5280-679X

Universidad de Piura, Dep. Ingeniería Industrial y de Sistemas Av. Ramón Múgica, 131. Apdo. postal 352, Piura, Perú alejandro.ruiz@udep.pe

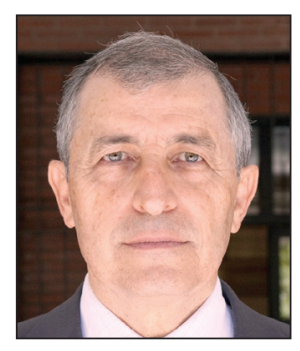

José-Antonio Moreiro-González es catedrático de biblioteconomía y Documentación de la Universidad Carlos III de Madrid. Es autor de trabajos sobre técnicas de análisis de contenido documental y cuestiones conceptuales de la documentación. Ha sido director del Departamento de Biblioteconomía y Documentación y decano de la Facultad de Humanidades, Comunicación y Documentación.

http://orcid.org/0000-0002-8827-158X

Univ. Carlos III de Madrid, Depto. de Biblioteconomía y Documentación C/ Madrid, 126. 28903 Getafe (Madrid), España jamore@bib.uc3m.es

\section{Resumen}

Se cuestiona el funcionamiento de los motores de búsqueda para la web semántica y si la visualización gráfica de los resultados mejora la recuperación. Se han analizado los tipos de consultas que se pueden realizar y las principales características de un buscador semántico. Se ha seleccionado una consulta y se ha ejecutado en buscadores semánticos de acceso online con la DBpedia. Además, se han estudiado las funciones básicas y deseables en un buscador de la web semántica y se han revisado algunos estudios sobre la evaluación de buscadores semánticos. Por último se analizan las soluciones gráficas para mostrar los resultados de algunos buscadores. Las principales conclusiones inciden en la idea de que las soluciones de visualización de los resultados de las consultas no cumplen las expectativas propuestas en la bibliografía. .

\section{Palabras clave}

Datos abiertos, Datos enlazados, Web semántica, Buscadores, Motores de búsqueda, Consultas, Visualización, Representaciones gráficas, Evaluación, Navegación conceptual, DBpedia. 
Title: Information visualization and retrieval in the semantic web

\begin{abstract}
The authors have questioned how the semantic web search engines work and if the graphical display of the search results improves the retrieval. The types of queries that can be performed, as well as the main features of a semantic search engine, have been analyzed. A query was selected and executed in semantic search engines with online access to DBpedia. The basic and desirable functionality in a semantic web search engine, and reviewed studies about the evaluation of semantic search engines have been studied. Finally, there have been analyzed the graphic solutions for displaying the results of some search engines. The main findings underlines the idea that graphical solutions to display query results do not meet the expectations proposed in the bibliography.
\end{abstract}

\title{
Keywords
}

Linked open data, LOD, Semantic web, Queries, Search engines, Visualization, Graphical display, Evaluation, Conceptual browsing, DBpedia.

Morato, Jorge; Sánchez-Cuadrado, Sonia; Ruiz-Robles, Alejandro; Moreiro-González, José-Antonio (2014). “Visualización y recuperación de información en la web semántica". El profesional de la información, mayo-junio, v. 23, n. 3, pp. 319-329.

http://dx.doi.org/10.3145/epi.2014.may.12

\section{Introducción}

El proyecto linked open data ( $L O D)$ se basa en la propuesta de Berners-Lee de publicar datos enlazados en la web mediante el uso de URIs, que permiten nombrar las entidades y establecer enlaces que relacionen recursos (Bizer; Heath; Berners-Lee, 2009). Esta información se formaliza con estándares como RDF, con sentencias en forma de tripletas con la secuencia recurso-atributo-valor. Posteriormente estas tripletas pueden ser interrogadas con lenguajes como Sparql, donde la potencia en la recuperación se produce por la combinación de tripletas de diferentes recursos.

En 2011 el proyecto LOD ya contaba con 295 datasets, con más de 31 millones de tripletas (Bauer; Kaltenböck, 2011) y diferentes recursos interrelacionados, caracterizándose por las enormes dimensiones de sus componentes. Por ejemplo FreeBase está formado por 43 millones de tópicos y 2,3 millones de hechos. DBpedia contiene 3,7 millones de tópicos, incluyendo 832.000 personas o 639.000 lugares.

http://dbpedia.org/About

http://www.freebase.com/browse

El elevado número de conceptos interrelacionados hace patente la necesidad e importancia de un método intuitivo para organizar, recuperar, filtrar, visualizar y navegar entre conceptos y acceder a sus recursos vinculados. En el estudio de Méndez y Greenberg (2012) se recogen y sintetizan diversas propuestas desde la perspectiva de $L O D$.

\section{Consulta y navegación en la web semántica}

\section{Tipos de consultas y respuestas}

Las consultas que caracterizan la web semántica consisten en la formulación de una única respuesta a una pregunta dada o a la obtención de un listado relacionado con una entidad, más que a una localización de palabras claves contenidas en documentos en lenguaje natural. Se interroga sobre un dato simple como una fecha, un nombre, un lugar (ele- mentos también conocidos como entidades del nombre), o bien sobre listados de entidades con atributos o facetas comunes que permitan filtrar la información. Según la propuesta clásica de Broader (2002), los tipos de consultas son:

- Informacionales: pueden ser tanto de instancias de tipos de datos (listado de músicos alemanes, fecha de las olimpiadas de Londres, etc.) como de refinamiento o filtrado de resultados por características, facetas, etc. (músicos alemanes del siglo XX con más de cinco sinfonías).

- Navegacionales: para, dado un recurso encontrar otros similares (webs sobre tecnologías para almacenar energía), o bien, dado un concepto encontrar documentos relacionados (sitios con fotografías de monumentos en París).

- Transaccionales: aquellas que permiten interactuar con el sitio una vez localizado.

La interrogación semántica implica conocer a priori dónde preguntar y cómo se representaron los conceptos y sus relaciones

Obviamente esta clasificación no está orientada, por ejemplo, a la localización de recursos de conocimiento formalizados (como ontologías, vocabularios de metadatos, etc.). Un ejemplo podría ser localizar un vocabulario de metadatos que incluya conceptos relacionados con un corpus de documentos. Ante estas carencias, investigadores como Uren et al. (2007) sugieren otros tipos de consultas como: consulta de entidad, relacional (que busca relaciones entre entidades) y parametrizada, que sería similar a las conocidas en documentación como facetadas. De forma análoga, Morato et al. (2013) identifican consultas conceptuales, contextuales y las que permiten el acceso a un recurso. También se han categorizado según las necesidades de información 
del usuario (Wei; Barnaghi; Bargiela, 2008; Strasunskas; Tomassen, 2010). Otras tipologías hacen hincapié en la formulación de la consulta: Fazzinga y Lukasiewicz (2010) clasificaron las consultas semánticas en estructuradas, con palabras clave y en lenguaje natural.

Los distintas tipos de consultas pueden devolver tipos de resultados diferentes: una consulta sobre un dato normalmente puede requerir una única tripleta; un listado puede precisar un conjunto de tripletas; y la petición de una o más páginas asociadas a un concepto, puede devolver una página enlazada mediante una o más tripletas. Por ejemplo, para obtener "un listado de municipios españoles y la población correspondiente a cada uno de ellos", se crea una consulta y los datos resultantes pueden proceder de varias combinaciones de tripletas.

\section{Tipos de buscadores semánticos}

Presentan una gran diversidad según sus características. Su interfaz de búsqueda puede presentar formularios, grafos o permitir el uso de un lenguaje de recuperación. Lopez et al. (2011) centraron la clasificación en aspectos como: el ámbito, el grado de estructuración de las fuentes, los tipos de datos para expresar la consulta y el contexto de búsqueda (desambiguación, multilingüismo, ranking o fiabilidad). Además, el buscador puede tener o no una ontología subyacente para navegar semánticamente entre los conceptos.

La satisfacción del usuario ante la complejidad de la web semántica no puede medirse con métricas típicas de recuperación de información

Algunos permiten búsquedas sólo contra un dataset o un vocabulario, mientras que otros permiten consultas federadas contra más de un conjunto de datos. También varían en cuanto a la usabilidad y conocimiento técnico requerido por el usuario. Por último cada buscador utiliza un lenguaje de interrogación, y una ontología que puede determinar la potencia y complejidad para realizar la consulta.

Algunas propuestas plantean buscadores similares a Google en cuanto a un formulario sencillo como interfaz de búsqueda. Es el caso de Swoogle, Sindice, Falcons o Dr.Watson, que

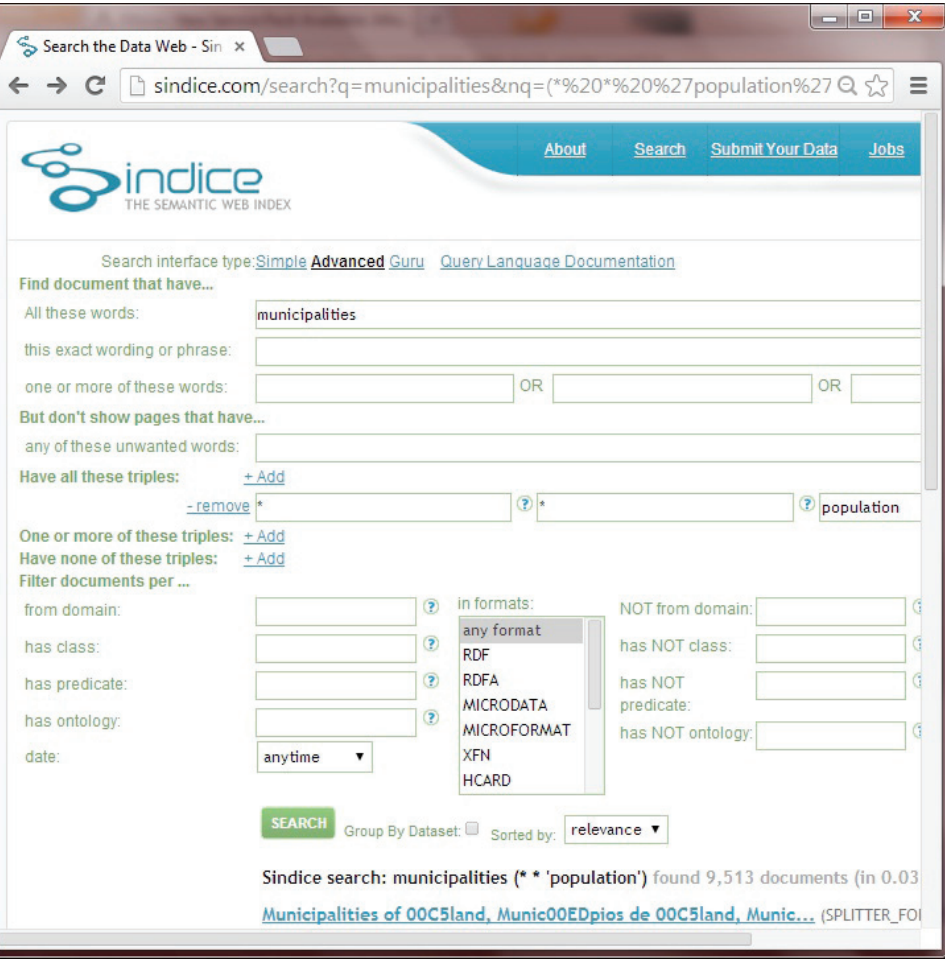

Figura 1. Búsqueda avanzada en Sindice para buscar la población de municipios. http://sindice.com presentan una interfaz despejada que permite una consulta sencilla basada en una palabra clave. Sin embargo, esta aparente usabilidad contrasta con los interrogantes planteados al usuario sobre cómo se ha representado cada concepto, como se observa en la figura 1, Lo que supone un conocimiento especializado previo sobre las consultas y las respuestas que se pueden obtener.

Estos buscadores proporcionan una solución parcial para resolver los tipos de consultas de la web semántica. Por una parte por la dificultad de interrogar al buscador, cuando se desconoce el recurso o si no se tiene un conocimiento de los lenguajes de la web semántica (Miličić, 2011; Morato et al., 2013), y por otra, por la dificultad añadida que supone la interpretación de los resultados, bien por el modo en el que se ordenan, o bien por cómo se muestra el resultado y su contexto.

En cuanto a las opciones de búsqueda y el lenguaje de interrogación para los buscadores semánticos, dependiendo del tipo de buscador y de consulta se exigen algunos conocimientos a los usuarios como:

- Conocer un recurso que contenga esta información.

- Conocer la estructura de conocimiento que defina el con-

Tabla 1. Comparación de las definiciones para el elemento "agent"

\begin{tabular}{|l|l|}
\hline \multicolumn{1}{|c|}{ Vocabulario } & \multicolumn{1}{c|}{ Definición elemento "agent" } \\
\hline $\begin{array}{l}\text { NSDL Registry } \\
\text { http://metadataregistry.org }\end{array}$ & The person or organization having a role with the vocabulary \\
\hline $\begin{array}{l}\text { MulDiCat } \\
\text { http://www.ifla.org }\end{array}$ & $\begin{array}{l}\text { A person (author, publisher, sculptor, editor, director, composer, etc.) or a group (family, organization, corpora- } \\
\text { tion, library, orchestra, country, federation, etc.) or an automaton that has a role in the lifecycle of a resource }\end{array}$ \\
\hline $\begin{array}{l}\text { FOAF } \\
\text { http://www.foaf-project.org }\end{array}$ & An agent (eg. person, group, software or physical artifact) \\
\hline $\begin{array}{l}\text { VCard } \\
\text { http://tools.ietf.org/html/rfc6350 }\end{array}$ & An entity who may sometimes act on behalf of the entity associated with the vCard \\
\hline
\end{tabular}




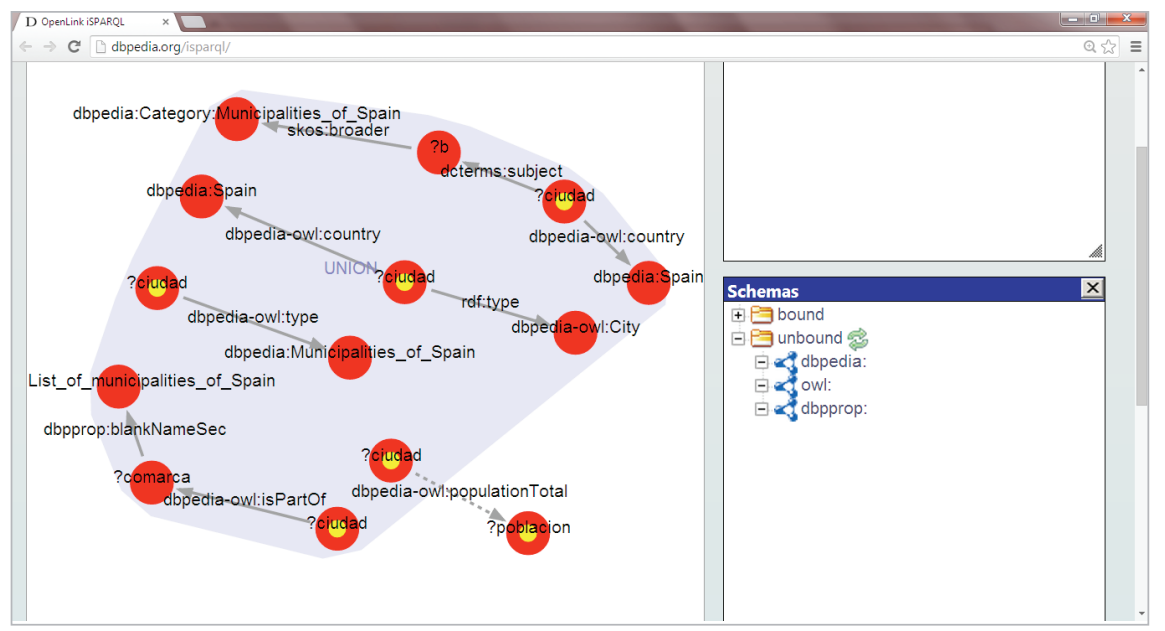

Figura 2. Grafos alternativos sobre población de municipios en España con iSparql http://dbpedia.org/isparql mos y comparamos los resultados se aprecia que el solapamiento de las definiciones es parcial (tabla 1), por lo que el concepto que representan podría no ser exactamente el mismo. http://lov.okfn.org/dataset/lov/index.html http://metadataregistry.org

Algunos trabajos, como el de Fuentes-Lorenzo, Morato y Gómez (2009), proponen mostrar el contexto en lenguaje natural y anotadores que conectados a vocabularios simplifiquen su codificación. No obstante, sin visualizar las relaciones del término, el problema persiste. cepto general en ese recurso y cómo está representado el dato. Un mismo concepto puede estar representado por criterios diferentes.

- Ser consciente de las diferencias de matices entre las definiciones alternativas referidas a la representación de un concepto. Esto es, una misma codificación puede representar conceptos más genéricos, más amplios o más específicos dependiendo del vocabulario.

- Para consultas complejas, conocer el lenguaje de interrogación del buscador (como Sparql).

- Valorar la consulta más eficaz en el caso de que existan alternativas.

En el siguiente punto se detallan ejemplos de los elementos discutidos en esta enumeración.

\section{Representación y codificación de los conceptos}

Un mismo concepto puede ser representado en distintos vocabularios o esquemas de metadatos con diferente terminología y codificado en diferentes lenguajes de representación, por eso se habla de representación y codificación de conceptos. Si se desconoce en qué vocabulario se encuentra el concepto, una aproximación razonable para realizar la consulta sería utilizar recursos que abarquen múltiples vocabularios como Open metadata registry $(O M R)$ o Linked open vocabularies (LOV). No obstante, nos encontraremos con los problemas asociados a la polisemia y falta de contexto. Por ejemplo en la búsqueda para el concepto "agent", el OMR lo encuentra en 4 vocabularios y LOV lo localiza en 88 . Si extrae-

\section{Formulación de la consulta}

Hemos desarrollado el ejemplo propuesto previamente sobre "un listado de municipios españoles y la población correspondiente a cada uno de ellos" en la DBpedia. En la tabla 2 observamos la formulación de consultas alternativas en Sparql, pero no equivalentes en su resultado. En el ejemplo las entidades buscadas pueden estar categorizadas como: Municipalities_of_Spain (consulta 1), una consulta más limitada con city con un valor de filtrado igual a Spain (consulta 2), ciudades que tengan el término genérico Municipalities_of_Spain (consulta 3), o una consulta equivalente a la tres para el caso de que haya una subdivisión administrativa como provincia o similar (consulta 4).

Se confirma que la formulación de esta consulta implica al menos tres dificultades:

- conocimiento de la sintaxis del lenguaje de búsqueda y la posibilidad de combinar tripletas y filtros;

- identificación de los conceptos sobre los que se han representado los datos;

- en caso de consultas alternativas, selección de la formulación con datos más confiables.

\section{Resultados de las consultas}

La figura 2 muestra los grafos resultado de las cuatro consultas alternativas sobre población de municipios en España del apartado anterior en OpenLink iSparql. Observamos como cada secuencia de nodos muestra la relación que los

Tabla 2. Consultas Sparql para recuperar la población de municipios: prefijos dbpedia: http://dbpedia.org/resource; dbpedia-owl: http://dbpedia.org/ontology; category: http://dbpedia.org/resource/Category; dbpprop: http://dbpedia.org/property

\begin{tabular}{|c|l|}
\hline 1 & $\begin{array}{l}\text { SELECT DISTINCT ?ciudad ?poblacion } \\
\text { WHERE \{\{?ciudad dbpedia-owl:type dbpedia:Municipalities_of_Spain.\} } \\
\text { OPTIONAL \{?ciudad dbpedia-owl:populationTotal ?poblacion.\}\} }\end{array}$ \\
\hline 2 & $\begin{array}{l}\text { SELECT DISTINCT ?ciudad ?poblacion } \\
\text { WHERE \{\{?ciudad rdf:type dbpedia-owl:City. ?ciudad dbpedia-owl:country dbpedia:Spain.\} } \\
\text { OPTIONAL \{?ciudad dbpedia-owl:populationTotal ?poblacion.\}\} }\end{array}$ \\
\hline 3 & $\begin{array}{l}\text { SELECT DISTINCT ?ciudad ?poblacion } \\
\text { WHERE \{\{?ciudad dbpedia-owl:country dbpedia:Spain. ?ciudad dcterms:subject ?b. ?b skos:broader category:Municipalities_of_Spain.\} } \\
\text { OPTIONAL \{?ciudad dbpedia-owl:populationTotal ?poblacion.\}\} }\end{array}$ \\
\hline 4 & $\begin{array}{l}\text { SELECT DISTINCT ?ciudad ?poblacion } \\
\text { WHERE \{\{?ciudad dbpedia-owl:isPartOf ?comarca. ?comarca dbpprop:blankNameSec dbpedia:List_of_municipalities_of_Spain.\} } \\
\text { OPTIONAL \{?ciudad dbpedia-owl:populationTotal ?poblacion.\}\} }\end{array}$ \\
\hline
\end{tabular}


une, y cada una de estas secuencias corresponde a una de las consultas realizadas en el apartado anterior.

Aunque todas las búsquedas devuelven resultados válidos, al compararlas se observa que no son equivalentes: no se obtienen los mismos datos en cuanto a nombres, número de municipios y población.

Los datos obtenidos se han comparado con los publicados por el Instituto Nacional de Estadística (INE) para 2013. El INE recoge 8.249 municipios en España. Se han considerado las variaciones ortográfica 0 idiomática. En la tabla 3 se observa el número de lugares recuperados y coincidentes con los recogidos en el INE. Se han calculado también las tasas de precisión y exhaustividad sin tener en cuenta el dato de población ni el año al que corresponde ese valor

Una observación más pormenorizada de los datos muestra que entre los resultados de las consultas 1 y 2 el porcentaje de ciudades coincidentes es sólo de un 3\% y aproximada-

Tabla 3. Exhaustividad y precisión respecto a los datos del INE http://www.ine.es

\begin{tabular}{|c|c|c|c|c|}
\hline Pregunta & $\begin{array}{c}\text { Coincidentes } \\
\text { INE } \\
\text { (T test 95\%) }\end{array}$ & Exhaustividad & Precisión & $\begin{array}{c}\text { Ciudades } \\
\text { encontradas } \\
\text { sin dato po- } \\
\text { blación (\%) }\end{array}$ \\
\hline 1 & $\begin{array}{c}1.442 \\
(p=0,022)\end{array}$ & 0,17 & 0,88 & 7 \\
\hline 2 & $\begin{array}{c}192 \\
(p=0,084)\end{array}$ & 0,02 & 0,85 & 1 \\
\hline 3 & $\begin{array}{c}4.911 \\
(p=0,003)\end{array}$ & 0,59 & 0,92 & 2 \\
\hline 4 & $\begin{array}{c}1.325 \\
(p=0,043)\end{array}$ & 0,16 & 0,86 & 3 \\
\hline
\end{tabular}

mente del $84 \%$ para las parejas 1 y 3 , y 3 y 4 . En el caso de ciudades coincidentes entre las consultas 2 y 3 es un $96 \%$. Por ejemplo Madrid sólo se obtiene en la consulta 3 y Barcelona se obtiene en todas salvo en la consulta 2. Los datos de las poblaciones coincidentes de la DBpedia y el INE son significativamente diferentes. Con estos datos se muestra que no todas las formulaciones tienen los mismos niveles de ruido y silencio en la recuperación.

A modo de ejemplo, en la figura 3 se muestran los resultados de la consulta 1 . En el listado completo se detectan ausencias significativas, incoherencias, e incluso datos erróneos. Otro ejemplo, que dificultaría las consultas relacionales (Uren et al., 2007) sería la consulta "Select ?a ?b Where \{?a skos:broader ?b. ?b skos:broader ?a.\}" que proporciona como resultado 1.566 conceptos cuyo genérico y específico es el mismo concepto.

Los principales problemas encontrados en la recuperación son:

- dificultad para formular consultas en el lenguaje de recuperación debido a su complejidad;

Tabla 4. Propuestas orientadas a mostrar de un modo gráfico las relaciones

\begin{tabular}{|c|c|c|}
\hline Propuestas & Principal función & Problema que afronta \\
\hline $\begin{array}{l}\text { VisualSparql y Welkin } \\
\text { http://graves.cl/visualSparql } \\
\text { http://simile.mit.edu/welkin }\end{array}$ & Visualizar gráficamente consultas Sparql & $\begin{array}{l}\text { Dificultad de uso del lenguaje de } \\
\text { recuperación }\end{array}$ \\
\hline Di-Martino (2010) & $\begin{array}{l}\text { Transformar consulta PLN en Sparql } \\
\text { y representarla gráficamente }\end{array}$ & $\begin{array}{l}\text { Dificultad de traducir el lenguaje natu- } \\
\text { ral al de consulta }\end{array}$ \\
\hline $\begin{array}{l}\text { Cypher } \\
\text { http://docs.neo4j.org/chunked/milestone }\end{array}$ & $\begin{array}{l}\text { Lenguaje de consulta gráfico con bases de } \\
\text { datos gráficas } N e 04 j\end{array}$ & $\begin{array}{l}\text { Dificultad de uso del lenguaje de } \\
\text { recuperación }\end{array}$ \\
\hline $\begin{array}{l}\text { Iconvis y Wikimindmap http://iconvis.polito.it/iconvis } \\
\text { http://WWW.wikimindmap.org }\end{array}$ & $\begin{array}{l}\text { Búsqueda gráfica secuencial y facetada de } \\
\text { entidades del nombre }\end{array}$ & $\begin{array}{l}\text { Problemas para restringir el ámbito de } \\
\text { búsqueda }\end{array}$ \\
\hline $\begin{array}{l}\text { Sgvizler } \\
\text { http://dev.data2000.no/sgvizler }\end{array}$ & $\begin{array}{l}\text { Resultados con distintos tipos de gráficos } \\
\text { (histogramas, dispersión, geolocalización, etc.) }\end{array}$ & $\begin{array}{l}\text { Escasa adaptación de los buscadores a } \\
\text { distintos tipos de consultas }\end{array}$ \\
\hline Mejía-Sánchez-Bermejo (2013) & $\begin{array}{l}\text { Desambiguación mediante el cálculo de la } \\
\text { distancia semántica en el grafo }\end{array}$ & $\begin{array}{l}\text { Problemas asociados a la denomina- } \\
\text { ción de un concepto en un vocabulario } \\
\text { debido a sinonimia y polisemia }\end{array}$ \\
\hline Thinkpedia y Thinkbase (Hirsch; Hosking; Grundy, 2009) & Visualización de vocabularios & $\begin{array}{l}\text { Dificultad de navegar debido a la abun- } \\
\text { dancia de conceptos }\end{array}$ \\
\hline
\end{tabular}




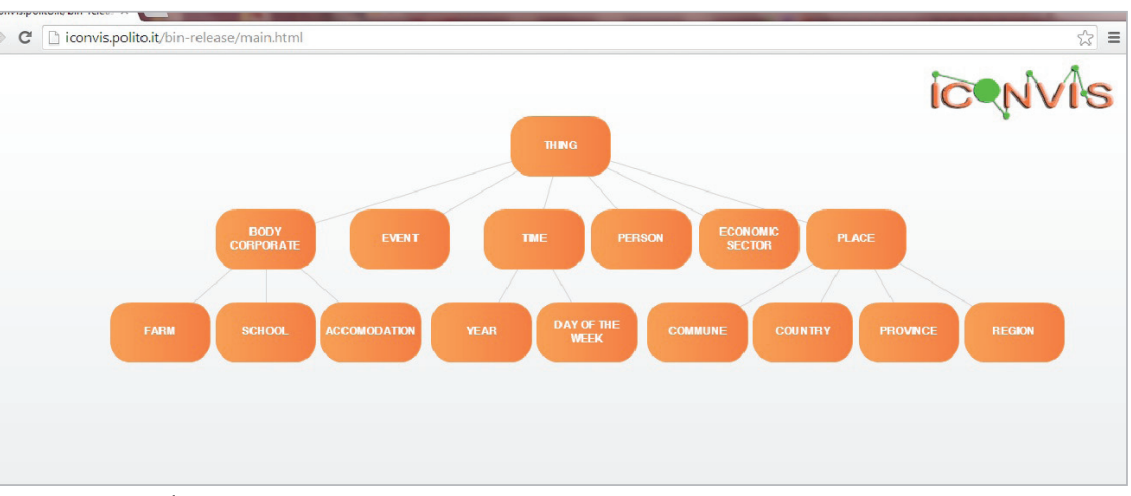

Figura 4. Consulta en Iconvis

http://iconvis.polito.it/iconvis

- posibles inconsistencias en las definiciones entre vocabularios al definir el mismo concepto;

- problemas asociados a la denominación de un concepto en un vocabulario debido a sinonimia y polisemia;

- dificultad para navegar debido al volumen de conceptos;

- dificultad para saber a qué vocabulario/s debemos interrogar;

- escasa usabilidad y funcionalidad de los buscadores de la web semántica a los distintos tipos de consultas y usuarios no expertos;

- problemática con moléculas RDF, es decir dificultad de expresar determinadas ideas en una única tripleta.

\section{Visualización y navegación web}

El volumen de datos que puede producir una consulta y la dificultad para interpretar los resultados ha dado lugar a iniciativas encaminadas a visualizar gráficamente los vocabularios, tratando de simplificar la consulta y contextualizando los conceptos. Hirsch, Hosking y Grundy (2009) señalan que la web social, la web semántica y las distintas técnicas de visualización de información pueden interactuar entre sí para simplificar y optimizar el proceso de consulta. Es decir, se trata de mejorar la visualización de los recursos de la web semántica, dado que esta visualización tiene como objetivo representar objetos de información de forma que el acceso a la información y al contenido subyacente sea más sencillo y comprensible (Baeza-Yates; RiveraLoaiza; Velasco, 2004). Dependiendo de la información, ésta podrá ser visualizada como un dato, una imagen, una tabla o un grafo.

La complejidad del lenguaje Sparq/ y las deficiencias en la visualización limitan la popularidad de los recursos semánticos

Algunos ejemplos de herramientas orientadas a la visualización y la navegación web son VisualSparql, Welkin, Iconvis, Cypher, entre otras muchas. Por ejemplo, Iconvis (figura 4) permite navegar entre nodos asociados de forma gráfica. Cada nodo se expande y muestra las clases y propiedades asociadas, convirtiendo el evento en un conjunto de consultas Sparql. Tras varios nodos se muestra el resultado en la Wikipedia.

La tabla 4 recopila propuestas que muestran de modo gráfico las relaciones entre conceptos enlazados semánticamente, y expone la función más relevante y las principales dificultades que conlleva su uso.

Tabla 5. Elementos para el análisis de sistemas de recuperación semántica

\begin{tabular}{|c|c|c|c|}
\hline Uren et al. (2007) & Mangold (2007) & Strasunskas y Tomassen (2010) & Morato et al. (2013) \\
\hline $\begin{array}{l}\text { Entorno de búsqueda: escala, hetero- } \\
\text { geneidad y portabilidad }\end{array}$ & Arquitectura & Arquitectura & \\
\hline Tipos de consulta & $\begin{array}{l}\text { Necesidades de informa- } \\
\text { ción del usuario }\end{array}$ & Objetivo de la búsqueda & Tipos de consulta \\
\hline \multirow[t]{5}{*}{$\begin{array}{l}\text { Posibilidad de realizar consultas de for- } \\
\text { ma iterativa y exploratoria: refinamien- } \\
\text { to, recomendación y reutilización }\end{array}$} & $\begin{array}{l}\text { Posibilidad de modifica- } \\
\text { ción de la consulta. Trans- } \\
\text { parencia e interactividad }\end{array}$ & $\begin{array}{l}\text { Expresión de búsqueda Input del } \\
\text { usuario (palabras clave, lenguaje } \\
\text { natural, gráficos, consulta formal e } \\
\text { interactiva) }\end{array}$ & \\
\hline & Estructura de la ontología & $\begin{array}{l}\text { Sistemas de organización del conoci- } \\
\text { miento }(K O S)\end{array}$ & $\begin{array}{l}\text { Gestión semántica: desambiguación, } \\
\text { multilingüismo, sinonimia, reuso }\end{array}$ \\
\hline & $\begin{array}{l}\text { Formalización de la } \\
\text { ontología }\end{array}$ & $\begin{array}{l}\text { Formalización de la ontología (RDFS, } \\
O W L, \ldots)\end{array}$ & $\begin{array}{l}\text { Lenguaje en que se expresa } \\
\text { Posibilidad de modificar el esquema } \\
\text { para mejorar la interoperabilidad, } \\
\text { formalización, interactividad }\end{array}$ \\
\hline & $\begin{array}{l}\text { Acoplamiento entre onto- } \\
\text { logía y documentos }\end{array}$ & & $\begin{array}{l}\text { Posibilidad de gestionar el marco } \\
\text { semántico y capacidad de modificar } \\
\text { ámbito, extensibilidad, modificabi- } \\
\text { lidad }\end{array}$ \\
\hline & & $\begin{array}{l}\text { Alcance (según sea aplicación de } \\
\text { escritorio o web) }\end{array}$ & \\
\hline $\begin{array}{l}\text { Problemas intrínsecos: Comprensión, } \\
\text { ranking de resultados y alineamiento }\end{array}$ & & & \\
\hline
\end{tabular}




\section{Criterios para la evaluación de buscadores semánticos}

Algunos autores sostienen que las medidas clásicas de precisión y exhaustividad son aplicables a la evaluación de motores semánticos de recuperación (Tümer; Shah; Bitirim, 2009; Andago; Phoebe; Thanoun, 2010). En general estos estudios no evalúan recursos de la web semántica, sino motores de documentos en lenguaje natural que aplican técnicas de extracción semántica y sistemas de organización del conocimiento.

Otros autores como Strasunskas y Tomassen (2010) señalan que la satisfacción del usuario al enfrentarse a la complejidad de una aplicación de un recuperador semántico no puede ser medida con estas métricas de recuperación de la información. La solución según estos autores es una evaluación que incluya aspectos cualitativos, como la calidad de los documentos formalizados, la calidad de la ontología subyacente, la arquitectura, usabilidad o la dificultad de la consulta. Diversos autores (Esmaili; Abolhassani, 2006; Uren et al., 2007; Mangold, 2007; Morato et al., 2013; Strasunskas; Tomassen, 2010) citan los siguientes elementos a evaluar:

- análisis de los buscadores según el tipo de consulta;

- calidad de los sistemas de organización de conocimiento (relaciones de dominio, meronimia, sinonimia, jerarquía, instancias y negación);

- similitud entre documentos y la ontología;

- criterios de indización de los documentos con la ontología;

- capacidad para representar, modificar o expandir la consulta o interactuar con el usuario;

- presentación o visualización de los resultados.

Muchos de estos factores son interdependientes, por ejemplo, los criterios de indización o la capacidad de expandir la consulta dependen de la riqueza semántica representada en el sistema de organización de conocimiento.

Como se puede observar en la tabla 5 , todos los autores consideran relevante los tipos de consulta en la evaluación. Por otro lado, algunos señalan la necesidad de incluir una evaluación de la interfaz de búsqueda (Strasunkas; Tomassen, 2010), mientras otros se centran en las características de la ontología subyacente (Morato et al., 2013). Hirsch, Hosking y Grundy (2009) mencionan que dada la diferente calidad de los recursos subyacentes, se dificultan las pruebas de usabilidad heurística en los usuarios. Como alternativa, estos investigadores proponen recopilar los comentarios de los usuarios al navegar por el recurso.

Existen variantes para representar el mismo concepto y formular la misma consulta

\section{Análisis de las interfaces gráficas de consulta semántica}

Vistos los problemas en la recuperación, se han analizado interfaces gráficas orientadas a facilitar la formulación de consultas semánticas. El objetivo es determinar en qué medida son una solución a algunos de los problemas discutidos. Se ha restringido el estudio a aquellas interfaces que pueden ser consultadas online sin precisar una instalación local (como es el caso de Allegrograph). También para evitar problemas derivados de la existencia de varias ontologías se ha limitado el estudio a aquellos recursos que operan con DBpedia.

Es necesario analizar los buscadores semánticos en escena- 


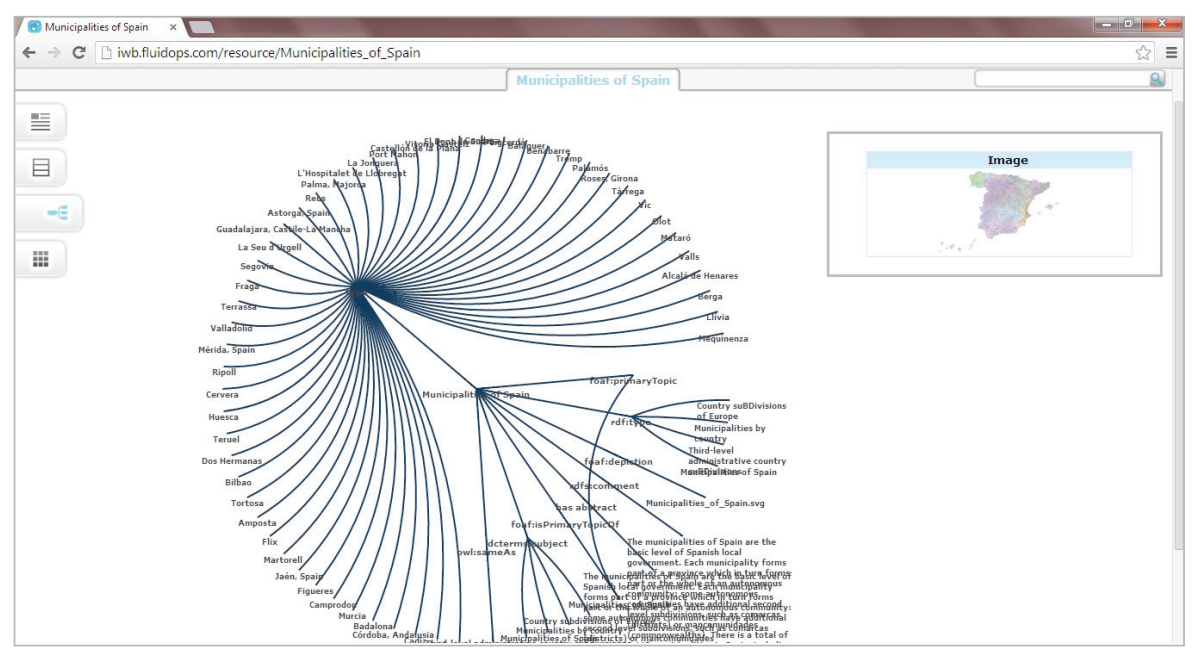

Figura 7. Linked open data demonstrator
-Desventajas: al tener sus funciones divididas entre varias herramientas su uso es más difícil. Además de la ambigüedad de los términos sugeridos, el abarrotamiento de términos, la dificultad de filtrado y tiempos excesivos complican su uso.

-Escenario: aunque ha sido una de las más completas y permite interrogar por varias tripletas $y$ elegir caminos alternativos, presenta problemas para filtrar o establecer un ranking sobre cuál es el camino más acertado.

\section{Linked open data demonstrator http://iwb.fluidops.com}

rios que incorporen elementos como interactividad, filtrado y diversos tipos de consulta. Se ha propuesto una tarea consistente en una ampliación de la consulta de ejemplo sobre municipios españoles, posteriormente refinando a los diez más poblados y accediendo a páginas web relacionadas. Señalando las principales ventajas y desventajas a partir de nuestra experiencia con el buscador. Finalmente se ha realizado una comparativa de algunos aspectos indicados en la tabla 5.

A continuación se analizan algunas de las características de los buscadores:

\section{Oobian}

http://dbpedia.oobian.com

Aplicación desarrollada por Maisis, compañía especializada en gestión del conocimiento y de contenidos. Es una de las que mejores resultados ha proporcionado para probar el escenario propuesto.

- Ventajas: posee una interfaz intuitiva. Permite refinar la consulta y la visualización es rápida, accediendo a recursos web relacionados.

- Desventajas: escasa capacidad de combinar entidades. La consulta Sparql generada no es visible.

- Escenario: presentó problemas para combinar entidades, descargar los listados, ordenar o filtrar resultados.
Es una plataforma para linked data, que integra varias fuentes (DBpedia, Twitter o Facebook). Permite integrarlo con otros recursos como big data.

- Ventajas: fácil de utilizar, y gráficos novedosos. Contiene recursos asociados a los términos. Fácil de consultar en lenguaje natural, pero no para todos los tipos de consultas. Es sencillo obtener un listado con las distintas tripletas a partir de un recurso.

- Desventajas: la visualización no es intuitiva, incluso la fuente llega a ser ilegible. Las consultas relacionales son complicadas de realizar.

- Escenario: las dificultades en interrogación simultánea por varios conceptos, obtención de listados y la gran densidad de conceptos dificultan su uso.

\section{Wikistalker}

http://sepans.com/wikistalker/\#

Muestra los enlaces de cada artículo de la Wikipedia.

- Ventajas: visualización en un solo vistazo de todos los enlaces del artículo, es de las pocas que posee un ranking visual de resultados.

- Desventajas: la visualización se resiente del elevado número de enlaces, no permite búsquedas más allá de las de un artículo.

\section{Visual dataweb relfinder}

http://www.visualdataweb.org/ relfinder/relfinder.php

La aplicación se subdivide en: Relfinder, especializado en buscar relaciones entre datos $R D F$, permitiendo acceder a nuevas tripletas. Utiliza Adobe Flex, lo cual le dota de mayor portabilidad. Por otro lado, GFacet permite una exploración a partir de cualquier elemento de la tripleta, con capacidad de filtros y de unir nuevos nodos.

- Ventajas: posibilidad de interrogar por varias tripletas. Muestra mucha información sobre las entidades interrogadas.

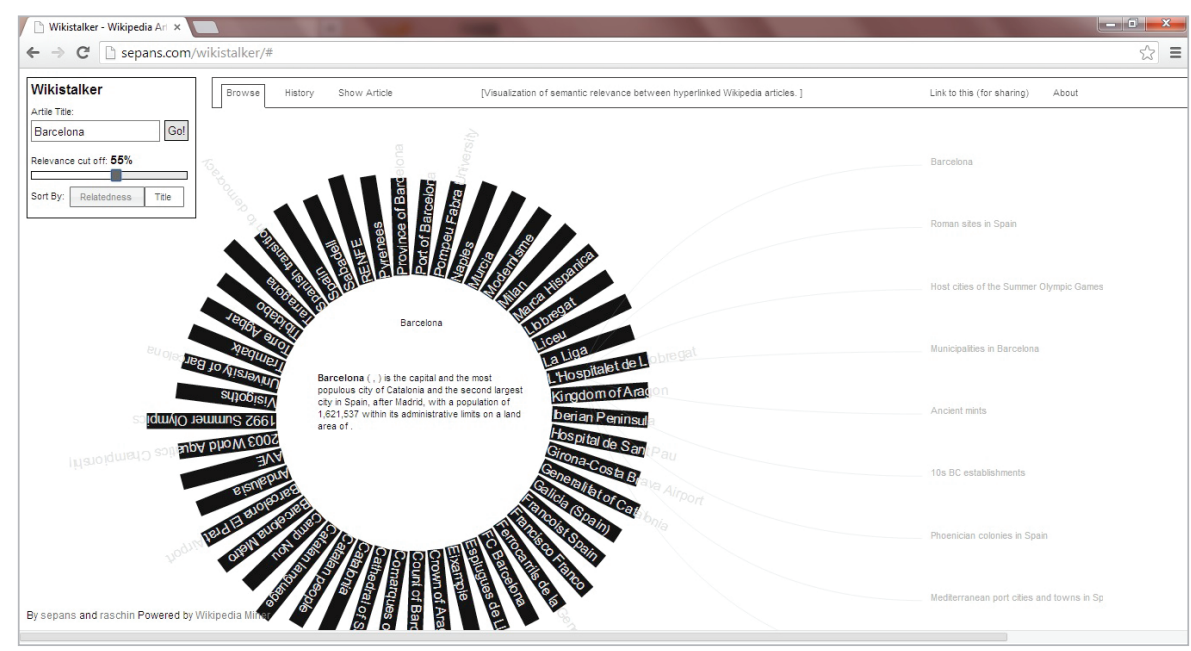

Figura 8. Wikistalker 
- Escenario: aunque indica enlaces relacionados, el acceso al documento o la descarga de los resultados de la búsqueda no es posible. Como en otros casos, el acceso a varias tripletas de forma simultánea no forma parte de las funciones del sistema.

\section{Lodlive}

\section{http://en.lodlive.it}

Se trata de una interfaz usable y funcional. Se basa en tecnologías de gran difusión y libres como Java Script, jQuery, html y JSON.

- Ventajas: facilidad para navegar entre resultados relacionados semánticamente.

- Desventajas: dificultad para consultar varias tripletas al mismo tiempo y acceder a los documentos de resultados.

- Escenario: la imposibilidad de combinar varias tripletas, descargar listados y documentos asociados y volumen de datos mostrados sin limitar el espacio de búsqueda hacen imposible la ejecución de las tareas.

\section{Las interfaces gráficas muestran grandes volúmenes de datos pero escasas posi- bilidades de filtrar y combinar consultas}

En la tabla 6, basada en la tabla 5, se analizan aspectos relacionados con los tipos de consulta, viendo la posibilidad gráfica de expansión de la consulta a elementos relacionados semánticamente, acceso documental a páginas web relacionadas y posibilidad de descargar elementos de las tripletas resultantes (como listas de entidades). El refinamiento (Uren et al., 2007; Mangold, 2007) se ha analizado observando la capacidad de buscar instancias en las que se solapan varios conceptos (ejemplo: solapamiento de conceptos población y municipio) y la posibilidad de filtrado (ejemplo: municipios con más población). Elementos como portabili-

unitérminos, mostrando listas de conceptos relacionados. Por último, se vio la posibilidad de realizar un ranking bien entre consultas alternativas o con los resultados, como propone Uren et al. (2007).

En algunos datos en la comparativa de la tabla 6, la implementación es parcial, así en Lodlive la consulta Sparql se puede introducir, pero no se puede obtener la conseguida con la navegación. Como conclusión se puede observar que la ausencia de ranking, de descarga de tripletas, de búsqueda de elementos concurrentes e integración con Sparql limitan estas herramientas. Por lo tanto el escenario propuesto no ha podido completarse dado el escaso soporte para filtrados y consultas a múltiples conceptos (sólo Visual dataweb lo soporta).

\section{Conclusiones}

El uso de los recursos semánticos depende de la disponibilidad de buscadores orientados a los usuarios. Para ello, los motores de la web semántica deben optar por simplificar la interfaz de recuperación y representar los recursos y/o los resultados de forma gráfica, con la presunción de que una visualización de los recursos interrelacionados mejora la interpretación de los datos.

Tras probar la ejecución en diferentes buscadores semánticos se ha comprobado que no es factible a día de hoy presdad (Uren et al., 2007), multilingüismo y desambiguación (Morato et al., 2013) y expresión de búsqueda (Strasunskas; Tomassen, 2010) se estudiaron mediante la posibilidad de desambiguación de homógrafos y visualización de la consulta generada en lenguaje Sparql o similar. Casi todas las herramientas permiten comenzar la consulta en lenguaje libre, pero con

Tabla 6. Comparación de herramientas para algunas funciones

\begin{tabular}{|l|c|c|c|c|c|}
\hline & Oograph & $\begin{array}{c}\text { Visual da- } \\
\text { taweb }\end{array}$ & $\begin{array}{c}\text { LOD de- } \\
\text { monstrator }\end{array}$ & Wikistalker & Lodlive \\
\hline Desambiguación homógrafos en la consulta & Sí & Parcial & Sí & Sí & Sí \\
\hline Multilingüismo & Sí & Sí & Sí & Sí & Sí \\
\hline Búsqueda con expansión a relacionados & Síl & Sí & No & No \\
\hline Búsqueda por varios conceptos concurrentes & No & Sí & No & No & No \\
\hline Filtrado de resultados & Parcial & No & No & No \\
\hline Ranking consultas o resultados & No & No & No & No & Sí \\
\hline Acceso documentos resultantes & Sí & Sí & No & No & No \\
\hline $\begin{array}{l}\text { Descarga tripletas resultantes (ejemplo: } \\
\text { listas) }\end{array}$ & No & No & No & No & Parcial \\
\hline Visualización de la consulta Sparql & No & No & No & No & \\
\hline
\end{tabular}


cindir del lenguaje de interrogación tipo Sparql. En cuanto a cómo se representan los datos, se obtienen resultados diferentes condicionados a la formulación de la consulta, que requiere conocer además los vocabularios de metadatos por los que está representado el recurso que se desea interrogar. Conocer la representación de los datos en los recursos también es una tarea ardua, porque aunque existe control de vocabulario en cada uno de los recursos mediante los vocabularios de metadatos, son muchos los conceptos similares pero representados de forma diferente. Existe por tanto control de vocabulario a nivel metadato, pero no al fusionarse con otros vocabularios, por lo que es necesario conocer previamente cómo están representados los datos antes de poder interrogarlos.

Aún se requieren elementos que faciliten la recuperación y comprensión de los datos y recursos recuperados

La consecuencia más inmediata es la complicación para usar el motor de búsqueda. En comparación con los buscadores de la web tradicional, quedan patentes las diferencias en cuanto al tipo de consulta (que está lejos de parecerse a una consulta por palabras clave), a los requisitos necesarios para formular la interrogación, y al tipo de recurso que se va a recuperar.

Estos buscadores difícilmente pueden ser evaluados sólo mediante métricas de precisión y exhaustividad. Creemos que deben añadirse características cualitativas asociadas a la usabilidad y funcionalidad. La capacidad de los buscadores para localizar determinada información dentro del gran volumen de documentos en la web semántica podría ser mejorada con algoritmos de posicionamiento para determinar los datasets o vocabularios más fiables.

La función de los buscadores visuales actuales se limita a mostrar los nodos directamente relacionados, y con la ejecución de las consultas complejas no se obtienen los resultados esperados. La visualización de los datos no es clara en aquellas consultas cuyos resultados son muy abundantes y con muchas relaciones. La navegación es compleja puesto que no existen directrices que orienten al usuario. Consideramos que aún se requieren elementos que faciliten la recuperación y comprensión de los datos y recursos recuperados.

\section{Agradecimientos}

Agradecemos la financiación recibida por el proyecto Almahisto (HAR2011-27540) del Mincin.

\section{Bibliografía}

Andago, Martin O.; Phoebe, The P. L.; Thanoun, Bassam A. M. (2010). "Evaluation of a semantic search engine against a keyword search engine using first 20 precision". International journal for the advancement of science \& arts, v. 1, n. 2, pp. 55-63.

http://www.ucsiuniversity.edu.my/cervie/pdf/ijasa/ paperV1N2IT3.pdf
Baeza-Yates, Ricardo; Rivera-Loaiza, Cuauhtémoc; Velasco, Javier (2004). "Arquitectura de la información y usabilidad en la web". El profesional de la información, mayo-junio, v. 13, n. 3, pp. 168-178.

http://www.elprofesionaldelainformacion.com/ contenidos/2004/mayo/1.pdf

Bauer, Florian; Kaltenböck, Martin (2011). Linked open data: the essentials. Viena: Semantic Web Company. ISBN: 9783902796059

http://www.semantic-web.at/LOD-TheEssentials.pdf

Bizer, Christian; Heath, Tom; Berners-Lee, Tim (2009). "Linked data-the story so far". International journal on semantic web and information systems, v. 5, n.3, pp. 1-22.

http://tomheath.com/papers/bizer-heath-berners-leeijswis-linked-data.pdf

http://dx.doi.org/10.4018/jswis.2009081901

Broader, Andrei (2002). "A taxonomy of web search". En: Sigir Forum, v. 36, n. 2, pp. 3-10.

http://www.cis.upenn.edu/ nenkova/Courses/cis430/p3broder.pdf

http://dx.doi.org/10.1145/792550.792552

Di-Martino, Beniamino (2010). "An approach to semantic information retrieval based on natural language query understanding". En: ICWE 2010 Workshop, pp. 211-222. http://dx.doi.org/10.1007/978-3-642-16985-4_19

Esmaili, Kyumars-Sheykh; Abolhassani, Hassan (2006). "A categorization scheme for semantic web search engines". En: Aiccsa, pp. 171-178.

http://dx.doi.org/10.1109/AICCSA.2006.205086

Fazzinga, Bettina; Lukasiewicz, Thomas (2010). "Semantic search on the web". Semantic web: interoperability, usability, aplicability, v. 1, n. 1-2, pp. 89-96.

http://www.semantic-web-journal.net/sites/default/files/ swj51.pdf http://dx.doi.org/10.3233/SW-2010-0023

Fuentes-Lorenzo, Damaris; Morato, Jorge; Gómez, JuanMiguel (2009). "Knowledge management in biomedical libraries: a semantic web approach". Information systems frontiers, v. 11, n. 4, pp. 471-480.

http://eprints.networks.imdea.org/111/1/Knowledge_ management_in_biomedical_libraries_a_semantic_web_ approach.pdf

http://dx.doi.org/10.1007/s10796-009-9159-y

Hirsch, Christian; Hosking, John; Grundy, John (2009). “Interactive visualization tools for exploring the semantic graph of large knowledge spaces". En: Workshop on visual interfaces to the social and the semantic web (Vissw2009).

http://ceur-ws.org/Vol-443/paper6.pdf

Lopez, Vanessa; Uren, Victoria; Sabou, Marta; Motta, Enrico (2011). "Is question answering fit for the semantic web? A survey". Semantic web: interoperability, usability, aplicability, n. 2, pp. 125-155.

http://www.semantic-web-journal.net/content/questionanswering-fit-semantic-web-survey http://dx.doi.org/10.3233/SW-2011-0041

Mangold, Christoph (2007). "A survey and classification of 
semantic search approaches". International journal of metadata, semantics and ontologies, v. 2, n. 1, pp. 23-34.

http://dee.srv1.eu/refs/files/1/65.pdf

http://dx.doi.org/10.1504/IJMSO.2007.015073

Mejía-Sánchez-Bermejo, Antonio (2013). Similitud semántica entre conceptos de Wikipedia. Trabajo fin de grado. Universidad Carlos III de Madrid.

http://hdl.handle.net/10016/17170

Méndez, Eva; Greenberg, Jane (2012). "Linked data for open vocabularies and HIVE's global framework". El profesional de la información, mayo-junio, v. 21, n. 3, pp. 236-244.

http://www.elprofesionaldelainformacion.com/ contenidos/2012/mayo/03_eng.pdf

http://dx.doi.org/10.3145/epi.2012.may.03

Miličić, Vuk (2011). "Introducing hypernotation an alternative to linked data". Bew citnames.

http://milicicvuk.com/blog/2011/11/17/introducinghypernotation-an-alternative-to-linked-data

Morato, Jorge; Sánchez-Cuadrado, Sonia; Dimou, Christos; Yadav, Divakar; Palacios, Vicente (2013). "Evaluation of semantic retrieval systems on the semantic web". Library hi tech, v. 31, n. 4, pp. 638-656.

http://dx.doi.org/10.1108/LHT-03-2013-0026
Strasunskas, Darijus; Tomassen, Stein (2010). "On variety of semantic search systems and their evaluation methods". En: Procs of the Intl conf on information management and evaluation, pp. 380-387.

Tümer, Duygu; Shah, Mohammad-Ahmed; Bitirim, Yiltan (2009). "An empirical evaluation on semantic search performance of keyword-based and semantic search engines: Google, Yahoo, MSN and Hakia". En: $4^{\text {th }}$ Int l conf on internet monitoring and protection (Icimp'09), pp. 51-55.

http://dx.doi.org/10.1109/ICIMP.2009.16

Uren, Victoria; Lei, Yuangui; Lopez, Vanessa; Liu, Haiming; Motta, Enrico; Giordanino, Marina (2007). "The usability of semantic search tools: a review". The knowledge engineering review, v. 22, n. 4, pp. 361-377.

http://oro.open.ac.uk/23501/1/download.pdf

http://dx.doi.org/10.1017/S0269888907001233

Wei, Wang; Barnaghi, Payam; Bargiela, Andrzej (2008). "Search with meanings: an overview of semantic search systems". International journal communications of SIWN, n. 3, pp. 76-82.

http://personal.ee.surrey.ac.uk/Personal/P.Barnaghi/doc/ siwn2008Survey.pdf

http://citeseerx.ist.psu.edu/viewdoc/summary?doi=10.1.1.324.2897

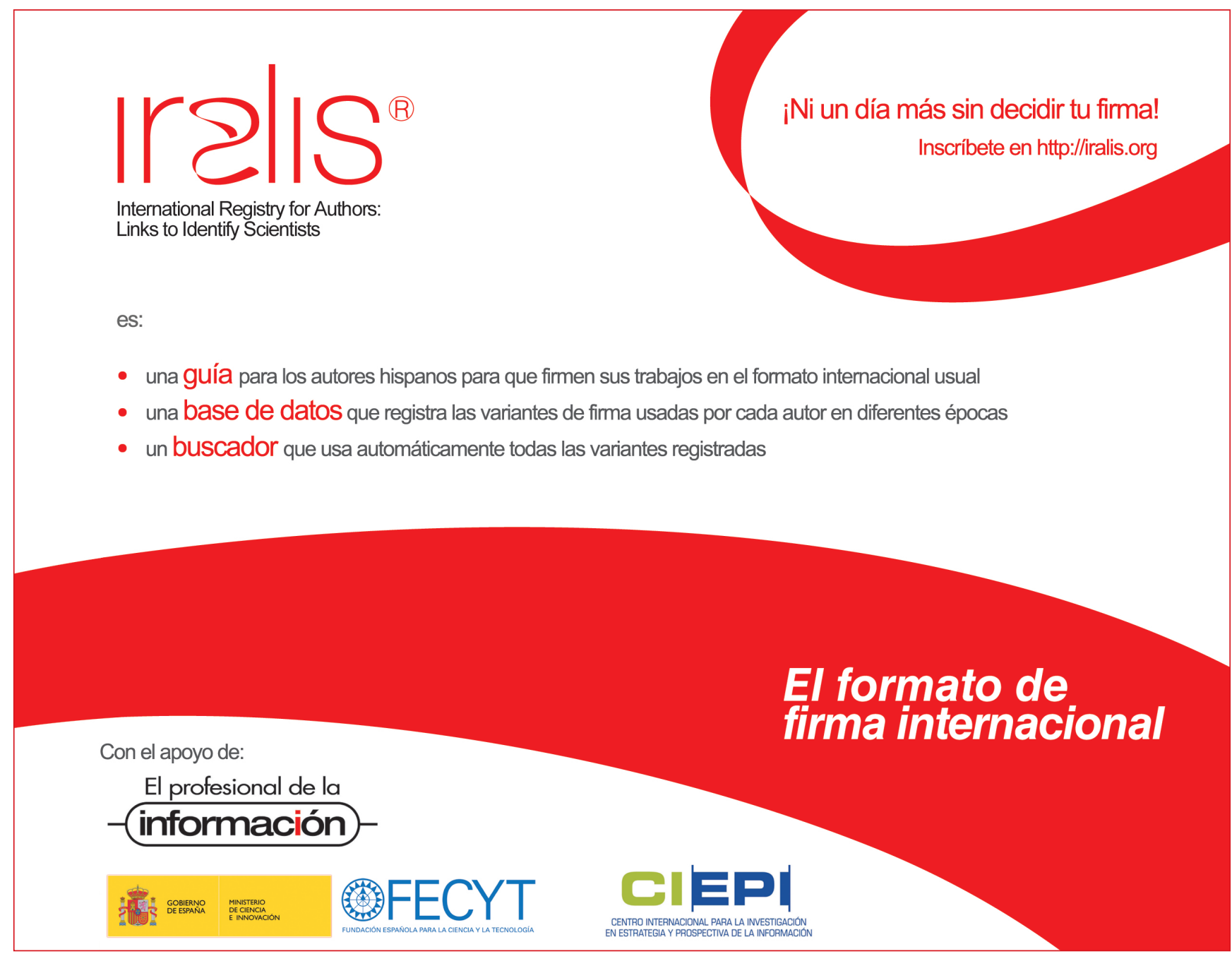

